Meta

Journal des traducteurs

Translators' Journal

\title{
Temps de créativité en traduction
}

\section{Mathilde Fontanet}

Volume 50, numéro 2, avril 2005

Processus et cheminements en traduction et interprétation

Processes and Pathways in Translation and Interpretation

URI : https://id.erudit.org/iderudit/010992ar

DOI : https://doi.org/10.7202/010992ar

Aller au sommaire du numéro

Éditeur(s)

Les Presses de l'Université de Montréal

ISSN

0026-0452 (imprimé)

1492-1421 (numérique)

Découvrir la revue

Citer cet article

Fontanet, M. (2005). Temps de créativité en traduction. Meta, 50(2), 432-447.

https://doi.org/10.7202/010992ar

\section{Résumé de l'article}

L'article a pour objet d'alimenter le débat sur la créativité dans le processus de traduction. Mon propos est d'illustrer par des exemples concrets que la créativité déployée pour résoudre des problèmes de traduction peut prendre des visages différents selon qu'elle contribue à l'aboutissement de la démarche de compréhension ou de la démarche de reformulation. 


\title{
Temps de créativité en traduction
}

\author{
MATHILDE FONTANET \\ Université de Genève, Genève, Suisse \\ mathilde.fontanet@cern.ch
}

\begin{abstract}
RÉSUMÉ
L'article a pour objet d'alimenter le débat sur la créativité dans le processus de traduction. Mon propos est d'illustrer par des exemples concrets que la créativité déployée pour résoudre des problèmes de traduction peut prendre des visages différents selon qu'elle contribue à l'aboutissement de la démarche de compréhension ou de la démarche de reformulation.
\end{abstract}

\section{ABSTRACT}

This article proposes to contribute to the discussion on creativity during the translation process. Using concrete examples, it aims to show that, when solving textual problems, a translator's creativity has various facets, depending on whether it contributes to the completion of the semasiological (comprehension) process or the onomasiological (reformulation) process.

\section{MOTS-CLÉS/KEYWORDS}

créativité, associations, pensée divergente, pensée latérale, problèmes de traduction

"Problems cannot be solved by thinking within the framework in which they were created."

Albert Einstein

\section{La problématique}

\subsection{Introduction}

C'est après une quinzaine d'années de pratique de la traduction, à l'occasion de mon initiation par Jeanne Dancette (2003) à l'exercice du "protocole de verbalisation", que j'ai décelé chez moi la présence de schémas comportementaux particuliers dans le traitement de certaines difficultés. Il est de fait apparu que, lorsque j'étais confrontée à un passage obscur, je commençais par explorer l'étendue sémantique de chacun des mots concourant à l'opacité du texte, puis j'abandonnais en général là ma réflexion pour traduire la suite, jusqu'à ce que, subitement, une solution me vienne comme spontanément à l'esprit. Intriguée par la récurrence du phénomène, j'ai décidé d'examiner un peu plus avant la manière dont j'aborde les difficultés de traduction.

\subsection{Objectifs et délimitation de la recherche}

J'ai donc entrepris de m'observer moi-même pour explorer comment, par mes ressources personnelles, je remédiais à des problèmes de reformulation ou à des insuffisances sémantiques du texte original et pour déterminer si l'opération comportait une dimension créative. Pour éviter que l'intervention de la créativité ne soit dictée 
par la nature du texte, je me suis limitée aux seuls textes administratifs, journalistiques et techniques, à l'exclusion des œuvres littéraires.

Ainsi, c'est moi-même que j'ai choisi comme objet d'observation. De ce fait, les réflexions qui suivent, loin de prétendre à démontrer quoi que ce soit, visent uniquement à présenter dans le détail la démarche d'une traductrice parmi d'autres, sans exclure ni présumer qu'il puisse s'agir d'un cas particulier. Mon propos n'est pas d'établir s'il existe un ou plusieurs types de traducteurs ${ }^{1}$ ou si des constantes seraient susceptibles de se dessiner dans le cadre d'une étude portant sur un nombre important de sujets. Je suis partie du principe que, quand bien même ma démarche traduisante relèverait tout à fait de l'exception, ma recherche pourrait présenter un intérêt, ne serait-ce qu'à titre comparatif.

Toute observation scientifique doit certes s'opérer dans des conditions aussi objectives que possible. Il m'est toutefois apparu que ma démarche trouverait une certaine légitimité dans la compréhension intime que l'observateur y aurait de son objet d'étude, une telle transparence étant difficilement envisageable dans le cadre d'une recherche portant sur une autre personne.

\subsection{Hypothèse de travail et définitions}

Je suis partie de l'hypothèse que le processus de traduction présente des moments de créativité distincts pour la résolution de problèmes de compréhension et pour le traitement des difficultés de reformulation.

Par problème de traduction, je désigne toute situation dans laquelle le traducteur se trouve dans l'incapacité de produire spontanément un équivalent du texte de départ en langue d'arrivée, dans la mesure où il prend conscience de cette incapacité et y remédie en passant par un processus de recherche de solution.

Par créativité, je désigne la capacité de « découvrir une solution nouvelle, originale, à un problème donné ${ }^{2}$.» Telle que je l'entends dans le contexte de la traduction, cette notion implique que le traducteur trouve une solution par ses propres ressources et non pas à la suite d'une recherche documentaire ou terminologique. À cette notion s'oppose celle d'automatisme, de réflexe et de mise à profit de l'expérience acquise ${ }^{3}$.

Je reprends ainsi à mon compte la définition de la solution créative que donne Weisberg, avant d'ajouter la notion de créativité dans la formulation du problème:

A creative solution to a problem must meet two criteria: it must be novel and it must solve the problem in question. [...] In well-defined problems, the characteristics of the solution are specified precisely at the beginning, so that the problem solver knows which criteria the solution must meet. [...] In addition to the creative solution to a problem, one can also talk about the creative analysis or formulation of a problem, which involves approaching a problem in a different way from the approaches taken by others. $(1986: 139 ; 142)$

\section{2. État de la question}

\subsection{Les études sur la créativité}

La créativité a fait l'objet de nombreuses études théoriques et empiriques, menées pour la plupart en psychologie ou dans des domaines connexes, en particulier la gestion et la prise de décisions ${ }^{4}$. 
Wallas (1926) est à l'origine de l'un des premiers modèles du processus de créativité. Comme avant lui le philosophe et mathématicien Henri Poincaré, il associe la créativité à un processus partiellement inconscient. Selon Wallas, pensée créative et pensée analytique sont complémentaires. Son modèle comporte quatre phases:

1) la préparation (définition, observation et étude du problème);

2) l'incubation (mise de côté du problème pour un certain temps);

3) l'illumination (émergence d'une idée nouvelle);

4) la vérification (contrôle).

Pour sa part, Rogers (1954: 300) définit trois caractéristiques des esprits créatifs:

1) l'ouverture à l'expérience (la curiosité, la sensibilité esthétique, ainsi que la capacité d'accepter des incohérences, des contradictions, l'inconnu, l'ambigu, l'approximatif et l'incertain);

2) une source interne d'évaluation (l'autonomie intellectuelle, la capacité de décider selon son propre jugement et d'évaluer selon ses propres critères, sans chercher à recevoir l'approbation d'autres personnes);

3) l'aptitude à jouer avec les concepts (la fluidité et la flexibilité conceptuelles, ainsi que l'aptitude à générer une grande quantité et une grande variété d'idées).

Koestler (1964) crée le terme de bissociation pour mieux définir la pensée créatrice et l'opposer à la pensée «routinière», qui ne s'exerce que sur un seul niveau. Selon lui, la créativité fait intervenir le rapprochement de concepts jusque-là non associés. Il dresse en 1976 une liste de critères opposant la routine et l'originalité.

\begin{tabular}{|l|l|}
\hline Aptitudes routinières (habitude) & Créativité (originalité) \\
\hline $\begin{array}{l}\text { association dans les limites d'un référentiel } \\
\text { bien défini }\end{array}$ & bissociation de référentiels indépendants \\
\hline $\begin{array}{l}\text { influence de processus préconscients ou non } \\
\text { conscients }\end{array}$ & influence de processus subconscients \\
\hline équilibre dynamique & activation de potentiels régénérateurs \\
\hline rigidité face aux variations & hyperflexibilité \\
\hline répétitivité & nouveauté \\
\hline attitude conservatrice & attitude destructivo-constructive \\
\hline
\end{tabular}

Deux notions importantes dans ce contexte aujourd'hui sont celles de «pensée divergente» et de "pensée latérale». La "production divergente» (divergent-production), qui opère sur un mode intuitif, s'oppose à la "pensée convergente», qui travaille sur un mode rationnel (voir Guilford 1967). La «pensée latérale» s'oppose quant à elle à la «pensée verticale», qui suit un mode logique (voir de Bono 1970).

Guilford et Höpfner (1971 : 186-187) définissent six facteurs de pensée créative: sensitivity to problems, fluency, flexibility, originality, redefinition et penetration.

Osborne (1974: 100-103) établit que «le procédé fondamental de [la] production d'idées, c'est l'association» et rappelle que les «anciens Grecs avaient posé les trois règles de l'association : la contiguïté, la similitude et le contraste. [...] L'association trouve son meilleur terrain chez ceux dont l'énergie imaginative est la plus intense et dont l'esprit est le plus meublé. Plus la mémoire est vive, mieux elle se prête au processus de l'association». "Notre production d'idées dépend du contenu de notre esprit ainsi que de la manière dont nous combinons ses ressources. Dans cette opération, l'association d'idées joue un rôle de catalyseur». 
Montuori et Purser (1995) estiment que les personnes créatives se caractérisent par leur tendance à se remettre en question et par leur perceptivité à leur environnement:

Creative individuals seek to understand their environment and are willing to put their own beliefs and assumptions into question in order to do so to a far greater degree than are persons who were not judged as being particularly creative. Creative persons are therefore constantly engaged in a process of self-renewal that draws on environmental factors for the destabilization of existing concepts, values, self-images and so forth.

Enfin, Gabora (2002) définit deux phases du processus créatif, qu'elle met en relation avec deux formes de pensée: l'une marquée par le mode suggestif, intuitif et associatif, l'autre par le mode convergent, analytique et appréciatif. Selon elle, l'esprit créatif passe d'un mode à l'autre selon les besoins.

The existence of two stages of the creative process is consistent with the widely-held view that there are two distinct forms of thought. The first is a suggestive, intuitive associative mode that reveals remote or subtle connections between items that are correlated but not necessarily causally related. This could yield a potential solution to a problem, though it may still be in a vague, unpolished form. The second form of thought is a focused, evaluative analytic mode, conducive to analyzing relationships of cause and effect. In this mode, one could work out the logistics of the solution and turn it into a form that is presentable to the world, and compatible with related knowledge or artefacts.

This suggests that creativity requires not just the capacity for both associative and analytic modes of thought, but also the ability to adjust the mode of thought to match the demands of the problem, and how far along one is in solving it.

\subsection{Les théories sur la créativité dans le domaine de la traduction}

\subsubsection{Réflexions sur la créativité}

Plusieurs théoriciens se sont penchés sur la question de la créativité en traduction. Leurs points de vue divergent cependant. Si certains, comme Mounin, opposent créativité à fidélité, d'autres, plus récemment (et selon moi plus judicieusement) la subordonne à la recherche d'une «plausibilité interindividuelle ${ }^{5}$ ». Qianyuan (1995: 138) postule que «la traduction est un acte essentiellement créateur au même titre que celui du peintre ou de l'écrivain. » Parmi diverses raisons, il invoque que «[1]es exigences posées à la traduction ne relèvent [...] ni d'un "nombre égal" ni d'un "ordre de grandeur égal" mais d'un "même poids" et d'un "même effet". Sans l'apport créatif du traducteur, aucune traduction ne saurait être conforme à ces attentes". Ballard (1997: 106) aboutit pour sa part à la conclusion que la créativité mérite d'être étudiée par la traductologie: «La créativité, comme la subjectivité, fait partie de l'opération de traduction et il faut intégrer ces éléments dans une démarche scientifique d'observation et d'exploration du phénomène, si l'on veut élaborer une traductologie réaliste et honnête.»

Plusieurs auteurs ont cherché à qualifier la nature de la créativité du traducteur. Wilss (1999: 2) commence par la remettre en cause:

A translation is always a reaction to an existing text (the source text), which forms the basis on which the translator operates. Where there is no source text, there can be no translation (target text). The translator cannot act autonomously. That is why the 
much-discussed notion of "creativity" in the translation process is ambivalent if not problematical, and the elevation of the (literary) translator to the level of a "co-author" seems justifiable only in exceptional cases.

Il développe ensuite la notion de «message dérivé» :

Translators do not commit their own ideas to paper, but reformulate the ideas given to them by the author of the original text. They do not plan, organize or express an authentic message, but a "derived message", in which three phases can be identified: preparation, formulation and evaluation of the translation. The decisive stage is that of formulation, the quality of which depends on the translator's experience and knowledge, on his/her (re)creativity, problem-solving skills, routine and expressive abilities, perhaps even on his/her ethical approach. Translators operate in a grey zone between light and darkness.

Enfin, dans le cadre de l'enseignement, Lee-Jahnke préconise «des principes pédagogiques qui incitent à la créativité» (2001: 259). La créativité est aussi l'un des trois paramètres qu'elle applique pour l'évaluation sommative. Dans son appréciation de la créativité, Lee-Jahnke prend notamment en compte les spécificités culturelles, les sociolectes, l'isotopie, le réseau sémantique, le registre de langue, les collocations, les métaphores, les jeux de mots, le style et les connotations (2001:267).

\subsection{2 Élaboration de modèles de créativité propres à la traduction}

Kussmaul, qui souscrit au processus de créativité en quatre phases défini par les chercheurs en psychologie (préparation, incubation, illumination et évaluation), consacre un chapitre intéressant à la créativité en traduction dans son livre Training the Translator (1995). Il avance l'idée (particulièrement intéressante dans le cadre de cet article) que la créativité intervient également dans le processus de compréhension:

We usually associate creativity with production. Thus within the field of language, saying things, writing and translating texts are typical creative activities. But what about comprehension? Is there not something like creative comprehension? We sometimes even talk of creative miscomprehension. [...] Comprehension, as we have seen now in many instances, is not only guided by what we hear or read but also by our personal knowledge and experience. Understanding is not merely a receptive but also a productive process. [...] It follows that when translating works of art creative understanding is part of the process. One might try to apply Guilford's notion of divergent thinking [...] to the comprehension process, and it would be interesting to see if these things can be observed in think-aloud protocols. For this purpose, however, one should perhaps use "more demanding" literary texts than the ones I have used so far. So at present, when observing creativity I have restricted myself to the reverbalization phase (Kussmaul 1995: 41).

Reprenant plusieurs idées de Guilford, il suggère que la qualité fondamentale en matière de créativité pour le traducteur est la fluidité (fluency), qui contribue à produire en peu de temps un grand nombre d'idées, d'associations et de pensées pour un problème donné. Il illustre aussi par divers exemples de protocoles de verbalisation l'efficacité de la divergent production. Par ailleurs, il souligne que le processus créatif, comme la plupart des activités mentales, n'est pas seulement régi par l'intellect, mais aussi par les émotions:

The creative process, however, as most mental activities, is not only governed by intellect but also by emotion. Some neurologists have put forward the hypothesis that creative 
thinking is closely connected with the anterior hypothalamus in the brain, which is the centre of libido and lust and motivates not only sexual fantasies but fantasies and daydreaming of all types. Such fantasies seem to be important for creative thinking (Kussmaul 1995: 48).

Selon lui, la confiance en soi et la conscience de soi (self-confidence et self-awareness) sont nécessaires pour une traduction féconde. Enfin, il estime qu'il est difficile de bien délimiter les diverses phases du processus de traduction (Kussmaul 2000: 76):

Wir haben die Illuminationsphase zusammen mit der Inkubationsphase beschrieben. Die Phasen gehen in der Wirklichkeit des kreativen Prozesses ineinander über. In der Kreativitätsforschung wird, wie schon gesagt, darauf aufmerksam gemacht, dass man generell damit rechnen muss, dass die einzelnen Phasen nicht streng chronologisch ablaufen, sondern dass es Vor- und Rückgriffe gibt, sozusagen gedankliche Schleifen, und dass manche Phasen mehrfach durchlaufen werden (vgl. Preiser 1976: 48). Die Phasen sind Denkmodelle, mehr oder weniger theoretische Konstruckte, um die komplexe Wirklichkeit in den Griff zu bekommen.

C'est également l'avis de Neubert (1997: 7), qui postule que les phases du processus de traduction s'interpénètrent:

The two stages of mediation are intricately intertwined. [...] For instance, what has been found to be truly significant in quite a number of think-aloud protocols (TAPS) is the occurrence of TL stances in the mind of the person involved in the ideation and partial anticipation of the written or spoken target text (Krings 1986, 1988; Lörscher 1991). Even more indicative of the mental activity of the mediator is the relatively strong grip on the translation process exerted by the wording of the source text (Schönfeld, 1995). Even during the production of the target text, fragmentary recollections of the source text, which translators or interpreters cannot escape the influence of, continue to influence target text production.

Enfin, Lörscher, considérant qu'aucun des modèles qu'il a étudiés ne permet de reconstituer de manière satisfaisante le processus psychologique de la traduction, conclut entre autres que:

[...] none of the models of the translation process can account for the psychological reality of translating. No conclusions can be drawn from the models as to what goes on in the head of a translator when s/he renders a source-language text into the targetlanguage. Nor do these models address the question whether the complex translation process consists of mental sub-processes, what their possible nature is, and how the translator integrates them during the process of translating. [...] The components of the translation process and their assumed interplay, as outlined in the models, have been construed rationalistically, by logical deduction, not by empirical induction. Thus, the translation process appears to be completely rational, which, however, it is only in ideality, but not in reality (Lörscher 1989: 64).

Lörscher préconise donc une investigation psycholinguistique du processus de traduction, fondée sur une étude empirique et sur "an analysis of translation performance». D’autres théoriciens mériteraient d'être cités ici, mais la taille de cet article ne le permet malheureusement pas. 


\section{Mes propres observations}

\subsection{Considérations générales}

Dans ma pratique de la traduction, j'ai pu observer que la meilleure stratégie à adopter pour parvenir à une bonne compréhension du texte de départ est de remettre en cause ses propres certitudes, sans jamais se fier aux évidences, et de rester perceptif aux contradictions du texte.

Selon moi, la phase de préparation comprend des démarches aussi diverses que le tâtonnement, la réflexion sur le contexte historique, le repérage des rapports formels ou sémantiques entre les mots du texte de départ, la consultation de dictionnaires ou d'autres ouvrages, la traduction provisoire, les interrogations, les paraphrases et les associations conscientes.

La phase d'incubation fait intervenir, entre autres, la temporisation, l'inscription de plusieurs solutions, les changements de perspective (mouvement pendulaire ou cyclique), la concentration sur d'autres problèmes, l'alternance entre considérations sémantiques et syntaxiques et les interrogations simultanées.

Enfin, l'activité traduisante exige une évaluation quasi permanente et une vérification systématique des solutions produites.

Comme il m'est apparu que je traitais différemment les difficultés de compréhension et les difficultés de reformulation, je les aborde ici séparément.

\subsection{Le traitement des difficultés liées à la compréhension du texte de départ}

\section{Exemple 1}

Lors de la traduction d'un procès-verbal d'une réunion, je me suis heurtée à la difficulté de saisir précisément la valeur de l'expression «deplorable record ${ }^{6} »$.

Mr X observed that his Government's formerly cordial relations with Cuba had come under considerable strain due to the latter's deplorable record on human rights.

\begin{tabular}{|l|l|}
\multicolumn{2}{|l}{ processus de compréhension } \\
\hline phase & processus \\
\hline préparation & $\begin{array}{l}\text { Perplexe, j'ai lu et relu la phrase en me concentrant sur le mot record, } \\
\text { cherchant à m'emplir de toute l'étendue de sa valeur sémantique (le passage } \\
\text { dans les annales, la consignation à un procès-verbal, la notion de dossier, de } \\
\text { casier judiciaire, de statistiques, etc.), sans m'arrêter sur un sens particulier. } \\
\text { Après être restée quelques secondes «dans le vague»,j'ai opté pour une } \\
\text { paraphrase dans l'attente d'une meilleure idée. }\end{array}$ \\
\hline incubation & $\begin{array}{l}\text { Laissant là le problème non résolu, j'ai entrepris de traduire la suite du texte. } \\
\text { À la fin du paragraphe, j'ai décidé de relire celui-ci. }\end{array}$ \\
\hline illumination & $\begin{array}{l}\text { Avant même de reprendre la phrase, les mots «notable», puis «notoire» me } \\
\text { sont venus à l'esprit, suivis, probablement par association, de l'expression } \\
\text { «inconduite notoire». }\end{array}$ \\
\hline vérification & $\begin{array}{l}\text { J'ai vérifié que la notion d'inconduite était bien compatible avec l'idée } \\
\text { exprimée par deplorable et que le mot «notoire» reprenait de manière } \\
\text { acceptable la valeur sémantique de record. }\end{array}$ \\
\hline
\end{tabular}


Une forme d'analyse combinatoire a probablement été à l'œuvre à l'arrière-plan de mon activité mentale tandis que je traduisais la fin du paragraphe: les diverses valeurs sémantiques que j'avais énoncées semblent avoir été successivement combinées avec les éléments du contexte, en produisant des solutions que ma conception de la plausibilité venait rejeter à mesure, jusqu'à ce qu'émerge une proposition satisfaisante. Une démarche purement rationnelle et déductive aurait probablement abouti au même résultat, mais, curieusement, ce n'est pas celle que j’ai adoptée. La traduction suivante a en fin de compte été retenue:

M. X indique que les relations cordiales qu'entretenait autrefois son gouvernement avec Cuba sont devenues très tendues du fait de l'inconduite notoire du pays en matière de respect des droits de l'homme.

\section{Exemple II}

Une nouvelle difficulté est survenue autour de la traduction de l'expression «which it was experiencing»:

Mrs X said that her country was naturally very aware of the gravity of the situation in Cuba, which it was experiencing at first hand through its embassy in Havana.

processus de compréhension

\begin{tabular}{|l|l|}
\hline phase & processus \\
\hline préparation & $\begin{array}{l}\text { Incertaine quant à la valeur d'experiencing (une forme d'expérience directe } \\
\text { paradoxalement acquise par la voie d'un intermédiaire), j'ai relu plusieurs } \\
\text { fois la phrase, atteignant presque un état d'hypnose. Rien ne semblait se } \\
\text { passer dans mon esprit. }\end{array}$ \\
\hline incubation & $\begin{array}{l}\text { J'ai opté pour la formule «qu'il a lui-même subi directement» dans l'attente } \\
\text { d'une meilleure solution et je suis passée à la suite du paragraphe. }\end{array}$ \\
\hline illumination & $\begin{array}{l}\text { Au moment d'attaquer la phrase suivante, l'expression «être témoin » m'est } \\
\text { spontanément venue à l'esprit. }\end{array}$ \\
\hline vérification & $\begin{array}{l}\text { Après avoir intégré l'expression dans le reste de la phrase, j'ai relu celle-ci } \\
\text { une ou deux fois, puis ai estimé que cette version était acceptable. }\end{array}$ \\
\hline
\end{tabular}

Je me suis tout d'abord laissé envoûter par le mot experiencing, me plongeant dans ses profondeurs, sans maîtriser le mouvement de ma pensée (qui semblait plutôt stagner). Au cours des instants d'absence qui ont suivi, j'imagine que diverses possibilités de traduction qui flottaient à mon insu dans mon esprit se sont polarisées par comptabilité sémantique et affinité formelle avec le microcontexte pour faire parvenir l'expression «être témoin» dans le champ de ma pensée. J'ai finalement retenu la formulation suivante:

Mme X déclare que son pays est naturellement bien conscient de la gravité de la situation à Cuba, dont son ambassade à La Havane est le témoin direct. 


\section{Exemple III}

J'ai ensuite été confrontée à la difficulté de comprendre le sens de l'expression selfcontained.

Mr B. stated that his delegation could support the Management's proposal, which it considered to be logical and self-contained.

\begin{tabular}{|l|l|}
\hline \multicolumn{2}{|c|}{ processus de compréhension } \\
\hline phase & processus \\
\hline préparation & $\begin{array}{l}\text { Je me suis arrêtée sur le mot self-contained, que je traduis souvent par } \\
\text { "autonome». J'ai laissé venir à mon esprit l'image d'une boîte et de son } \\
\text { contenu, de même que des associations comme «structure intérieure», } \\
\text { «qui se contient elle-même», «indépendante», «autosuffisante », «n } \\
\text { harmonie avec soi » et «d'une certaine maturité». Pour ne pas trop } \\
\text { m'attarder sur le passage, j'ai écrit «raisonnable» à titre provisoire et suis } \\
\text { passée à la suite. }\end{array}$ \\
\hline incubation & $\begin{array}{l}\text { J'ai traduit quelques paragraphes, puis, me rappelant qu'un problème était } \\
\text { resté en suspens, je suis revenue sur le passage. }\end{array}$ \\
\hline illumination & $\begin{array}{l}\text { J'ai relu l'original, puis ma traduction provisoire et le mot «cohérente» } \\
\text { m'est alors spontanément venu à l'esprit. }\end{array}$ \\
\hline vérification & $\begin{array}{l}\text { L'auteur du procès-verbal étant l'un de mes collègues, je lui ai demandé ce } \\
\text { qu'il avait voulu dire. D'abord perplexe, il s'est soudain rappelé que M. B } \\
\text { était l'un des rares délégués à s'exprimer en français lors de la réunion. } \\
\text { Comme mon collègue disposait d'un verbatim des diverses interventions, il } \\
\text { a pu m'indiquer que les mots qu'avait prononcés M. B. étaient en fait «qui } \\
\text { tient debout». }\end{array}$ \\
\hline
\end{tabular}

La préparation a consisté à étendre autant que possible le champ des possibilités de traduction de l'expression concernée en «mettant à plat» tout ce qui s'y associait dans mon esprit. Lors de l'incubation, un balayage inconscient de cette étendue s'est probablement opéré en quête d'une solution compatible avec le contexte. J'ai retenu la traduction suivante:

M. B. déclare que sa délégation peut appuyer la proposition de la Direction, qu'elle considère comme logique et cohérente.

\section{Exemple IV}

La difficulté suivante, qui porte sur la dernière phrase du passage retranscrit, a été rencontrée lors de la traduction d'une procédure de sécurité.

\section{Specific fire hazards}

[...] Another critical aspect is the possibility of the fire spreading behind or along the panels (through the fillings and joints) and propagating outside the structure. This is often in a difficult position for the Fire Brigade to reach or is out of sight. 
processus de compréhension

\begin{tabular}{|l|l|}
\hline phase & processus \\
\hline préparation & $\begin{array}{l}\text { J'ai relu la phrase et l'ensemble du paragraphe en m'interrogeant sur le sens } \\
\text { de This. }\end{array}$ \\
\hline incubation & $\begin{array}{l}\text { Tandis que je me laissais absorber par le texte m'est venue l'image de } \\
\text { pompiers voulant intervenir et cherchant la source de l'incendie. }\end{array}$ \\
\hline illumination & $\begin{array}{l}\text { Tout à coup, il m'a semblé évident que les pompiers voulaient voir par où se } \\
\text { propage le feu. }\end{array}$ \\
\hline vérification & $\begin{array}{l}\text { J'ai vérifié que la grammaire n'excluait pas cette interprétation et que c'était } \\
\text { bien la plus plausible et la plus logique dans le contexte. }\end{array}$ \\
\hline
\end{tabular}

On aurait pu s'attendre à ce que je m'engage dans une réflexion logique pour déduire le sens du contexte et de mes connaissances encyclopédiques - comme le font peut-être d'autres traducteurs. Ma démarche a cependant été différente: la solution semble m'avoir été suggérée par les images qui me sont venues à l'esprit. J'ai donc traduit le passage comme suit:

\section{Risques d'incendie particuliers}

[...] Un autre aspect critique est que le feu peut se propager derrière les panneaux ou le long de ceux-ci (en suivant la voie des colmatages ou des joints) et se transmettre à l'extérieur de la structure. Ces voies de transmission sont souvent invisibles ou difficiles d'accès pour les pompiers.

\subsection{Le traitement des difficultés liées à la reformulation}

\section{Exemple I}

Selon moi, la traduction du passage suivant, tiré d'un rapport sur les ressources humaines, a sollicité un bref processus créatif.

\section{Recruitment and retention}

More highly qualified levels in crucial skills have dominated recruitment in recent years and will continue to do so. However, this is becoming more and more difficult due to the increasing shortage of qualified candidates on the employment market.

processus de reformulation

\begin{tabular}{|l|l|}
\hline phase & processus \\
\hline préparation & $\begin{array}{l}\text { Après avoir traduit increasing shortage par «insuffisance croissante », j'ai } \\
\text { trouvé l'expression paradoxale et constaté une répétition de l'adjectif } \\
\text { insuffisant dans le paragraphe. Gênée, j'ai lu et relu la phrase anglaise sans } \\
\text { trouver de solution, puis je suis passée à la suite du texte. }\end{array}$ \\
\hline incubation & J'ai traduit quelques phrases, puis je suis revenue sur le passage. \\
\hline illumination & $\begin{array}{l}\text { En relisant ma traduction, le mot «raréfaction » s'est spontanément imposé } \\
\text { à moi. }\end{array}$ \\
\hline vérification & $\begin{array}{l}\text { J'ai relu ma traduction et l'ai comparée avec la version précédente pour me } \\
\text { convaincre qu'il s'agissait bien d'une amélioration. }\end{array}$ \\
\hline
\end{tabular}

Cette correction mineure semble m’avoir été inspirée directement par la langue, par le simple jeu des attirances et des répulsions entre les mots. Voici la solution retenue: 


\section{Recrutement et fidélisation du personnel}

Des niveaux de qualification plus élevés dans les domaines techniques clés ont caractérisé le recrutement ces dernières années et il en ira de même à l'avenir, malgré les difficultés croissantes qu'entraine la raréfaction des candidats qualifiés sur le marché de l'emploi.

\section{Exemple II}

J'ai été confrontée à un problème de reformulation lors de la traduction du titre d'un article devant paraître dans un bulletin interne.

\begin{tabular}{|c|c|}
\hline \multicolumn{2}{|c|}{$\begin{array}{l}\text { New Year, New People } \\
\text { "This is a bit like an Easter egg hunt", says Sam Wyse, his nose buried deep in the site } \\
\text { buildings map. He has to find building } 8 \text {, office } 9 \text {, in which the secretaries of the GP } \\
\text { division are waiting for him to reimburse him for his travelling expense. "Alright, it's between } \\
\text { building } 3 \text { and building } 4 \text {, so I have to turn left in the middle of building } 4 . . . \text { " he concludes } \\
\text { from the map. }\end{array}$} \\
\hline \multicolumn{2}{|c|}{ processus de reformulation } \\
\hline phase & processus \\
\hline préparation & $\begin{array}{l}\text { Après avoir constaté la présence d'un effet d'écho dans le titre, j'ai parcouru } \\
\text { l'article, qui traitait de la difficulté de s'orienter dans les bâtiments pour les } \\
\text { nouveaux membres du personnel. }\end{array}$ \\
\hline incubation & $\begin{array}{l}\text { J'ai entamé la traduction du texte sans plus penser au titre, en espérant } \\
\text { qu'une solution viendrait d'elle-même. }\end{array}$ \\
\hline illumination & $\begin{array}{l}\text { Tout à coup, le titre «Les tribulations d'un Chinois en Chine» m'est venu à } \\
\text { l'esprit. }\end{array}$ \\
\hline vérification & $\begin{array}{l}\text { Compte tenu que la moyenne d'âge des lecteurs de l'article allait être } \\
\text { relativement élevée, j'ai estimé qu'ils saisiraient une allusion au roman de } \\
\text { Jules Verne. }\end{array}$ \\
\hline
\end{tabular}

Ma démarche peut s'assimiler à une pensée divergente contrôlée, puisque j'ai donné libre cours à mon imagination après avoir défini les critères auxquels la solution devrait répondre (pour la forme: user d'une répétition; pour le fond: évoquer les mésaventures des nouveaux venus). La traduction que j'ai produite est la suivante:

\section{Les tribulations des nouveaux Cernois au CERN}

"C'est un peu comme chercher les œufs de Pâques», explique Sam Wyse, le nez plongé dans son plan des bâtiments du domaine. Il doit trouver le bâtiment 8 pour se présenter au bureau 9, où l'attendent les secrétaires de la Division GP pour lui rembourser ses frais de voyage. «Bon, c'est entre le bâtiment 3 et le bâtiment $4 \ldots$ il suffit donc de tourner à gauche au milieu du bâtiment 4 ...» conclut-il du plan.

\section{Exemple III}

La traduction du titre d'un article du bulletin du CERN m'a confrontée à la nécessité de reproduire un jeu de mots.

\section{The Proton Synchrotron flexes its ABS}

To most people ABS stands for Advanced Braking System, but to the PS control group, it's come to mean something quite different over recent years. ABS is the name given to a new set of control software for the accelerators in the PS complex, and the initials stand for Automated Beam Steering and shaping. 
processus de reformulation

\begin{tabular}{|l|l|}
\hline phase & processus \\
\hline préparation & $\begin{array}{l}\text { J'ai constaté la présence du jeu de mots dans le titre et, après avoir lu } \\
\text { l'article, j'ai estimé que la seule exigence était de jouer avec le sigle ABS. }\end{array}$ \\
\hline incubation & $\begin{array}{l}\text { J'ai commencé à traduire l'article. Par association, j'ai songé que le } \\
\text { contraire du freinage était l'accélération. Or quasiment toutes les activités } \\
\text { du CERN gravitent autour de l'accélération de particules. }\end{array}$ \\
\hline illumination & $\begin{array}{l}\text { Tout à coup, il m'est apparu qu'il suffisait d'opposer les activités de freinage } \\
\text { et d'accélération autour du sigle ABS. }\end{array}$ \\
\hline vérification & J'ai relu ma traduction, qui m'a paru suffisamment claire. \\
\hline
\end{tabular}

Le processus suivi dans ce cas s'apparente au précédent: des critères ont tout d'abord été définis, puis la solution semble être tombée du ciel. Il est fort probable qu'une forme de pensée divergente contrôlée ait été à l'œuvre. La traduction que j'ai choisie est la suivante:

\section{Au PS, l'ABS sert à accélérer!}

Le sigle ABS, bien connu pour signifier Advanced Braking System (système de freinage antiblocage), a pris un tout autre sens ces dernières années pour le groupe contrôlecommande du PS: c'est le nom d'un nouvel ensemble de logiciels de gestion des accélérateurs de l'ensemble PS, les initiales étant celles de Automated Beam Steering and shaping (aiguillage et mise en forme automatiques du faisceau).

\section{Exemple IV}

Le titre du paragraphe suivant (tiré d'un texte sur la Réforme de Bologne) m'a arrêtée dans un premier temps.

Kein Parallelismus: Ein Nebeneinander der bisherigen Diplom- respektiv Lizenziats-

Studiengänge und der neuen, gestuften Studiengänge (mit dem Bachelor als erstem und dem Master als zweitem Abschluss) im gleichen Fach ist zu vermeiden.

processus de reformulation

\begin{tabular}{|l|l|}
\hline phase & processus \\
\hline préparation & $\begin{array}{l}\text { Après avoir buté sur le sens du titre, j’ai lu le paragraphe et en ai conclu } \\
\text { que l'idée était de ne pas appliquer deux systèmes parallèlement. « Pas de } \\
\text { parallélisme» m’a toutefois semblé prêter à confusion. }\end{array}$ \\
\hline incubation & J'ai traduit le paragraphe. \\
\hline illumination & $\begin{array}{l}\text { La formule « un seul régime en vigueur» m'est venue naturellement à } \\
\text { l'esprit lorsque je suis revenue sur le titre. }\end{array}$ \\
\hline vérification & Après relecture, j'ai estimé que cette solution était satisfaisante. \\
\hline
\end{tabular}

Comme j'avais prédéfini le sens qu'il convenait d'annoncer, la recherche qui s'est opérée comme automatiquement a bien répondu à une forme de "cahier des charges ». J'ai probablement été conduite par association de l'absence de dualité (évoquée dans le texte) à la notion de «un seul». La traduction est restée la suivante:

Un seul régime en vigueur: Il convient d'éviter la coexistence, dans un même département, du régime actuel (prévoyant l'obtention d'un diplôme ou d'une licence) et du régime des filières d'études échelonnées (prévoyant l'obtention d'abord du bachelor, puis du master). 


\subsection{Les émotions liées au processus créatif}

Lors de la phase de préparation, j'éprouve soit une forme de curiosité enthousiaste, soit une certaine anxiété à la perspective de ne pas trouver de solution.

Pour que la phase d'incubation se déroule de manière satisfaisante, il est impératif que je sois détendue. Sous l'effet du stress, ma pensée s'enraie ou tourne douloureusement en rond: j'ai alors beau me concentrer sur les mots, lire et relire des phrases ou tenter de "passer à autre chose», rien ne se produit.

Dans la phase d'illumination, j'éprouve à la fois de la surprise et de la joie. Cette joie, qui est à la mesure de la difficulté du problème et de l'adéquation de la solution, semble de triple nature: intellectuelle, esthétique et existentielle. Je connais tout à la fois la satisfaction intellectuelle de résoudre un problème, le plaisir esthétique, quasi sensuel, de trouver une forme harmonieuse et, enfin, une joie existentielle à «laisser une trace », à penser que moi, traductrice, personnage de l'ombre, ai momentanément échappé à mon insubstantialité pour figer quelque chose de moi-même dans le texte.

Laukkanen (1996: 270) postule que des facteurs affectifs influencent le processus décisionnel de la traduction:

[A]ffective and attitudinal factors should not be ignored when translation processes are scrutinized. It seems worthwhile to do further research on both practising professional translators' and translation students' performance to find out, for example, how attitudes could and perhaps should be influenced during translator training.

De nombreux psychologues ont établi que le stress fait obstacle à la créativité. Ma propre expérience tend à confirmer que, lorsqu'il est préoccupé, obnubilé par les exigences qui lui sont imposées, le traducteur ne peut s'abandonner à la pensée divergente.

Dans la vie professionnelle (et dans le cadre des études), j'ai pu constater que certains réviseurs (et enseignants) déstabilisent les traducteurs (et étudiants) au point de leur faire perdre toute créativité. Or le processus de traduction, dès lors qu'il est confiné aux phases de préparation et de vérification, perd non seulement de son sel, mais aussi de son efficacité.

Selon Osborn (1974: 263-266), qui s'est intéressé à «la nature émotionnelle des impulsions internes », «la coercition ne peut que paralyser l'imagination». Au sujet de la peur, il estime que «les idées produites sous son influence sont mises en action sans recourir à l'évaluation ». Par ailleurs, «lorsqu'une passion violente prend possession de notre être, notre imagination a une tendance excessive à s'exalter et à négliger tout jugement». Enfin, "des expériences psychologiques ont confirmé le principe selon lequel la plupart des gens réfléchissent mieux lorsqu'ils ne sont pas soumis à une pression excessive».

\subsection{Commentaires sur les résultats}

Dans les exemples qui précèdent, les démarches sémiasologique et onomasiologique comprennent toutes deux une phase non contrôlée par l'esprit analytique, assimilable à une forme de pensée latérale ou de production divergente, avant que n'émergent des propositions, puis que celles-ci soient rigoureusement évaluées pour être soit sélectionnées, soit rejetées. 
Face à une difficulté de compréhension, je commence par me pénétrer de tous les sens potentiels du passage concerné, en me concentrant successivement sur chacun des mots et sur leur charge dénotative, connotative et associative avant de laisser mon esprit traiter inconsciemment ces informations (en les recombinant sous diverses formes) jusqu'à ce qu'il produise des propositions. Ces propositions, absurdes pour certaines puisqu'elles sont le fruit d'une pensée de type associatif, sont alors soumises à une évaluation rigoureuse (s'articulant autour des critères d'équivalence standard) jusqu'à ce qu'une solution satisfaisante soit trouvée.

Dans la phase de préparation, pour mieux «fouiller» toutes les facettes des unités sémantiques, il me faut "lâcher la bride» et recenser, en faisant taire mon sens critique, tout ce qui se présente à mon esprit comme associé aux mots du texte. Ce moment de réflexion, parce qu'il part du texte, mais passe par toutes les évocations qu'il fait naître en moi, prend un caractère autoréflexif. Lors de l'incubation, les propositions semblent germer dans le champ sémantique préalablement esquissé et se développer de long de l'axe de plausibilité défini par le contexte, comme s'il s'opérait une forme d'inférence inconsciente.

En revanche, lorsque la difficulté réside dans la reformulation en langue cible d'un sens bien perçu, je n'amorce ma « recherche d'inspiration » qu'après avoir défini les critères auxquels devra répondre ma traduction. Je laisse alors dériver ma pensée selon les mouvements qui lui sont propres, et dont le principe m'échappe, jusqu'à ce qu'elle produise des propositions, qui sont évaluées à mesure sur la base des critères préalablement établis. La pensée divergente peut soit m'accaparer pleinement, soit passer en quelque sorte au second plan, pour s'exercer parallèlement à mon traitement de la suite du texte.

C'est là assurément un aspect complexe du traitement des difficultés. Alors que je suis absorbée dans la perception de la suite du texte, que je cherche donc à le comprendre intellectuellement, mais aussi à le ressentir dans toutes ses aspérités, tout son volume, toutes ses nuances formelles et suggestives, il semble bien que la recherche par pensée divergente se poursuive en moi, puisque c'est bien souvent alors qu'émerge la solution du problème rencontré précédemment. Cela revient à dire qu'il est possible de traiter, à différents niveaux de conscience, plusieurs problèmes simultanément.

De même, lorsque je suis aux prises avec un problème de reformulation, deux mouvements antagonistes de la pensée semblent simultanément à l'œuvre: tandis que ma pensée dérive tous azimuts à la recherche de l'inspiration, chaque solution qui me vient à l'esprit est rigoureusement examinée. Le flot ininterrompu de la pensée évocatoire et associative semble filtré à mesure par mon esprit critique, selon des critères d'acceptabilité formelle et sémantique.

Au cours du processus de traduction, le sens ne semble pas nécessairement faire escale dans un no language's land comme le suggère la théorie de la déverbalisation. Il transiterait plutôt par un espace mental dans lequel est brassé, sous forme déstructurée, tout ce que le traducteur a «perçu» dans le texte, une forme de bouillon de sens, de mots, d'associations, d'émotions et d'évocations.

Compte tenu de la nature des diverses phases du processus de créativité, il me semble que c'est sur la préparation et la vérification que devrait porter l'enseignement de la traduction. Pour optimiser la phase d'incubation, c'est, comme le préconise Kussmaul, ladite fluency qu'il convient de renforcer. Or la fluidité se développe selon 
moi essentiellement par la lecture et d'autres activités extérieures à l'exercice de la traduction.

\section{Conclusion}

L'observation de ma propre pratique de la traduction tend à confirmer que le processus de résolution de problème peut s'opérer différemment selon que la difficulté relève de la compréhension ou de la reformulation du texte, même s'il intervient dans les deux cas une phase de pensée latérale ou divergente au cours de laquelle l'esprit s'engage dans une dérive associative en quête de solutions. Dans la démarche sémasiologique, la pensée divergente s'appuie sur une pluralité de sens possibles, qu'elle va librement combiner avec les éléments du contexte jusqu'à faire émerger une solution plausible, qui paraît inférée des données fournies au départ. Dans la démarche onomasiologique, en revanche, des critères sont définis avant de donner libre cours à la pensée divergente et le processus est essentiellement associatif.

\section{NOTES}

1. Tout comme il existe des gauchers et des droitiers, des séquentiels et des simultanés, des visuels et des auditifs.

2. Cette définition correspond à l'acception socio-psychologique du terme dans le Trésor de la langue française informatisé (voir $<$ http://atilf.atilf.fr/tlf.htm $>$ ).

3. À titre d'exemple, si un traducteur se trouve perplexe devant l'expression «There was a large consensus that...» et qu'il opte après un temps de réflexion pour la formule «De l'avis général...», solution de son propre cru, il fait selon moi preuve de créativité. En revanche, s'il recourt à la même solution par la suite, il ne s'agit plus de créativité, mais de réflexe ou d'expérience.

4. Pour une présentation générale des théories sur la créativité, voir l'article de Niska (1998), dont je me suis inspirée pour le survol des principaux modèles, de même que celui de Plsek (1996), qui présente une intéressante rétrospective de la question depuis 1908.

5. Pour une présentation générale de la pensée traductologique sur la créativité, voir Balacescu et Stefanink (2003).

6. La plupart des observations qui suivent ont été consignées à mesure que je me suis trouvée confrontée à des problèmes de traduction. D'autres ont été reconstituées de mémoire a posteriori.

\section{RÉFÉRENCES}

Balacescu, J. et B. Stefanink (2003) : «Modèles explicatifs de la créativité en traduction », Meta 48-4, p. 509-525.

Ballard, M. (1997): «Créativité et traduction», Target 9-1, J. Amsterdam, B.V. Benjamins, p. $85-110$.

Dancette, J. (1994): Parcours de traduction, Étude expérimentale du processus de traduction, Lille, Presses universitaires de Lille.

Dancette, J. (2003) : «Le protocole de verbalisation: un outil d'autoformation en traduction», La formation à la traduction professionnelle, Ottawa. P.U.G., Mareschal et al.

De Bono, E. (1970) : Lateral Thinking: A Textbook of Creativity, London, Ward Lock Educational.

Gabora, L. (2002) : «Cognitive Mechanisms Underlying the Creative process», Proceedings of the Fourth International Conference on Creativity and Cognition, October 13-16, Loughborough University, UK, p. 126-133 (voir aussi sous <http://www.vub.ac.be/CLEA/liane/papers/CandC/ CandC.htm>).

Guillford, J. P. (1967): The Nature of Human Intelligence, New York, McGraw-Hill, Inc.

Guillford, J. P. and R. Höpgner (1971): The Analysis of Intelligence, New York, McGraw-Hill Book Company. 
Koestler, A. (1976) : «Bisociation in Creation», Rothenberg \& Hausman, p. 108-113; réimpression de Koestler, Arthur (1964): The Act of Creation, London, Hutchinson Press.

Kussmaul, P. (1995): Training the Translator, Amsterdam, John Benjamins.

Kussmaul, P. (2000) : «Kreatives Übersetzen », Studien zur Translation, 10, Tübingen, Stauffenburg Verlag.

LaukKanen, J. (1996): "Affective and Attitudinal Factors in Translation Processes», Target 8, Amsterdam, John Benjamins B.V., p. 257-274.

Lee-Janhke, H. (2001): «Aspects pédagogiques de l'évaluation des traductions», Meta 46-2, p. 258-271.

Lörscher, W. (1989): «Models of the Translation Process, Claim and Reality», Target 1-1, p. 43-68.

Montuori, A., and R. E. Purser (1995): «Deconstructing the Lone Genius Myth: Toward a Contextual View of Creativity», Journal of Humanistic Psychology 35, p. 69-112.

Neubert, A. (1997): «Postulates for a Theory of Translation», Cognitive Processes in Translation and Interpreting, Applied Psychlogy, 3, Danks Joseph H. et editors, London, Thousand Oaks, p. 1-24.

Niska, H. (1998) : Explorations in Translational Creativity: Strategies for Interpreting Neologisms, Workshop paper, Stockholm University, <http://lisa.tolk.su.se/kreeng2.htm>.

Osborne, A. (1974): L'imagination constructive: créativité et brainstorming, traduit de l'ouvrage «Applied imagination» par G. Rona et P. Dupont, Paris-Bruxelles-Montréal, Dunod.

Quianyuan, G. (1995): «Das Wesen des Übersetzens ist kreativ», Babel, 41-3, Fédération internationale des traducteurs, p. 129-139.

Plsek, P. E. (1996): Models for the Creative Process, E. Plsek \& Associates, <http://www. directedcreativity.com/pages/WPModels.html>.

Rogers, Carl R. (1976) : «Toward a Theory of Creativity», Rothenberg \& Hausman, p. 296-305; réimpression (1954), A Review of General Semantics, ETC.

Wallas, G. (1926): The Art of Thought, New York, Harcourt Brace.

Weisberg, R. W. (1986): Creativity: Genius and Other Myths, New York, W. H. Freeman and Company.

WiLss, W. (1999): Translation and Interpreting in the 20th Century: Focus on German, Amsterdam/ Philadelphia, Benjamins Translation Library 29, John Benjamins Publishing Company. 